\title{
Estrategias metodológicas interactivas para la enseñanza y aprendizaje en la educación superior
}

\author{
Fecha de recepción : 2020-01-15 • Fecha de aceptación: 2020-05-10 • Fecha de publicación: 2020-09-10
}

\author{
María de los Ángeles Bonilla ${ }^{1}$ \\ Universidad Estatal de Bolívar \\ mabonilla@ueb.edu.ec \\ https://orcid.org/0000-0003-2051-4626 \\ Jonathan Patricio Cárdenas Benavides ${ }^{2}$ \\ Universidad Estatal de Bolívar \\ jcardenas@ueb.edu.ec \\ https://orcid.org/0000-0002-9760-602X \\ Florcita Janeth Arellano Espinoza ${ }^{3}$ \\ Universidad Estatal de Bolívar \\ farellano@ueb.edu.ec \\ https://orcid.org/0000-0002-4325-9317 \\ Danny Fernando Pérez Castillo 4 \\ Universidad Estatal de Bolívar \\ dperez@ueb.edu.ec \\ https://orcid.org/0000-0003-4418-0050
}

\section{Resumen}

Las estrategias metodológicas que imparten los docentes en el área académica de educación superior intervienen en la enseñanza y aprendizaje de los estudiantes. El objetivo del presente artículo se centra en analizar mediante una revisión bibliográfica documental las estrategias metodológicas interactivas más aplicadas y sugeridas en la educación superior. Con un enfoque cualitativo documental se ha revisado 15 artículos de los cuales 6 causaron más impacto en la investigación, a través de una matriz de contraste se determinaron 15 estrategias metodológicas interactivas con mayor rele- 
vancia hasta la actualidad. Se pudo concluir una de las tácticas que más se destacan son role play, caso de estudio, aprendizaje personalizado, discusiones y el E-learning, métodos que se generan en función de los módulos y necesidades de enseñanza, tomando en cuenta que gran parte del aprendizaje universitario es de modalidad autónoma; no obstante, la guía de un docente es el punto más importante a considerar, es por eso que se destacan tanto actividades individuales como estrategias de formación grupal.

Pallabras clave: aprendizaje, estrategias metodológicas, enseñanza, métodos.

\begin{abstract}
The methodological strategies taught by teachers in the academic area of higher education are involved in the teaching and learning of students. The objective of this article focuses on analyzing through a documentary bibliographic review of the most applied and suggested interactive methodological strategies. With a qualitative documentary approach, 15 articles have been reviewed, of which 6 were analyzed that caused the most impact on the investigation, through a contrast matrix, 15 more relevant interactive methodological strategies were determined to date for higher education. It was concluded that among the strategies that stand out the most are role play, case study, personalized learning, discussions and E-learning, strategies that are generated according to the modules and teaching needs, taking into account that much of the learning University is autonomous, however, the guidance of a teacher is the most important point to consider, which is why both individual activities and group training strategies stand out.
\end{abstract}

Keywords: learning, methodological strategies, teaching, methods. 


\section{Introducción}

Las estrategias metodológicas de enseñanza y de aprendizaje son herramientas que contribuyen a obtener resultados, son herramientas que buscan que la enseñanza se convierta en diferentes acciones interactivas, en sustento a la experiencia socio-didáctica de enseñanza, se comprende como el aporte de los elementos práctico-pedagógicos que se ejecutan en el proceso de interacción entre docentes y alumnos para crear ambientes de enseñanza y aprendizajes significativos en el ámbito educativo.

En relación con lo establecido, la apreciación didáctica mediante el enfoque por competencias permite reclutar estrategias didácticas de enseñanza y aprendizaje que permitan una pedagogía innovadora y cambiante en la práctica. Este proceso metodológico parte de la reflexión y el análisis del contexto contenido entre lo social y cultural para conocer y articular los elementos involucrados, el aprendizaje se fusiona a los elementos que rodean al estudiante en cualquier circunstancia tanto en el espacio físico como el aspecto social (Gutiérrez et al., 2018).

En el proceso de enseñanza efectuado con el enfoque por competencias, las secuencias o acciones didácticas inician a partir de la exploración de los aprendizajes iniciales que poseen los estudiantes, por lo tanto, estos aspectos se vinculan con la realidad social del escenario de aprendizaje, en donde el docente debe implementar su creatividad en el proceso didáctico e innovador para contextualizar sus actividades de enseñanza.

El enfoque basado en la competencia del sistema de educación superior está destinado a aumentar la atención a la formación efectiva y tecnológica de las competencias profesionales (Petruta, 2013). La competencia profesional la entendemos como una educación personal que determina la productividad de las tareas profesionales e incluye conocimientos, habilidades y cualidades personales significativas desde el punto de vista profesional, experiencias y orientaciones de valor. En este caso, la competencia difiere de conceptos tradicionales como conocimiento, habilidad, y experiencia por su naturaleza integradora, determinada por rasgos personales, como la práctica, enfoque y la capacidad de trabajar en una amplia variedad de contextos, autorregulación y autoestima (Yakovleva \& Yakovlev, 2014).

Dicha definición requiere cambios significativos en el apoyo pedagógico del plan de estudios universitario, llenándolo con métodos de enseñanza que podrían proporcionar la formación de futuros especialistas con el resultado integral requerido (Granjeiro, 2019). Los métodos tradicionales del proceso educativo universitario (conferencia, explicación, ejercicio, etc.) son ciertamente importantes para el desarrollo profesional; sin embargo, sus limitaciones se sienten aún más en la actualidad cuando se forma un fenómeno complejo como la competencia.

Por lo tanto, creemos que la educación moderna debe centrarse en la actividad independiente del alumno, la organización de entornos de autoaprendizaje y la formación experimental y práctica, donde los alumnos tienen una elección de acciones y pueden utilizar iniciativas (Carrera \& Marín, 2011). La práctica o aplicación de métodos de enseñanza innovadores e interactivos en las instituciones educativas tienen el potencial no solo de mejorar la calidad de la educación, sino también empoderar a los estudiantes o la generación futura del país fortaleciendo gobernanza y 
galvanizar el esfuerzo para lograr el objetivo de desarrollo humano para el país (Wickramsinghe, 2016).

Existen métodos de aprendizaje que se adaptan a las personas, que tienen un lugar en línea, en donde el aprendizaje incluye al E-learning, aprendizaje en Internet, aprendizaje distribuido, en red, tele-aprendizaje, de manera virtual, asistido por computadora, basado en la web y aprendizaje a distancia (Karmakar \& Nath, 2014). Es importante considerar a los métodos y formas de enseñanza con el fin de organizarlos para alcanzar un mayor aprovechamiento de capacidades cognitivas de los estudiantes, preparándolos para adquirir conocimientos por sí solos y así puedan ser aplicados de manera más práctica, original y creativa (Ledesma \& Labrador, 2010).

La disponibilidad del capital humano se concentra en un proceso global de actividades, ingenio y know how de los maestros que conlleven con esfuerzos internos fundamentales para obtener el logro de la estrategia de excelentes prácticas de manejo, utilización de plataformas virtuales, zoom, páginas web, entre otras tecnologías aplicadas por la emergencia sanitaria del COVID-19, con el propósito de que instruyan al estudiante con una excelente estrategia metodológica recibida previamente por el docente (Mora et al., 2018).

En función a lo descrito por Jurado \& Munoz (2017) una metodología paralela en el contexto de la educación superior es el involucramiento de los estudiantes hacia un trabajo de calidad en el momento en que el alumno esté completamente integrado en el proceso de acuerdo con sus capacidades y potencialidades. Las estrategias son mucho más que acciones grupales, promueven a que trabajen siguiendo sus intereses personales (Bonilla, Hallo, Quizhpe, \& Taco, 2020).

Estos mecanismos de enseñanza han evolucionado progresivamente con el paso del tiempo, en correspondencia con la necesidad educativa, buscando aportar suficientes soluciones para alcanzar un aprendizaje con calidad, rapidez, eficiencia (Lara, A., Lara, N., Lara, G., \& Bonilla, 2018).

Una estrategia metodológica es la proyección de un sistema de acciones a corto, mediano y largo plazo, las cuales permiten la dirección de enseñanza aprendizaje, tomando como base varios métodos y procedimientos para el logro de los objetivos definidos en un tiempo determinado. Tiene como finalidad promover la formación y desarrollo de estrategias de aprendizaje en los estudiantes.

Por otro lado, las estrategias de aprendizaje son el conjunto de actividades, técnicas y medios que se planifican de acuerdo con las necesidades de los estudiantes, los objetivos que se buscan y la naturaleza de los conocimientos, con la finalidad de hacer efectivo el proceso de aprendizaje.

Esto conlleva al proceso de enseñanza-aprendizaje que coordina la formación social de las nuevas generaciones, de tal forma que este proceso, dado su carácter integral, se convierte en un instrumento fundamental para satisfacer el encargo curricular, que en verdad es un problema ya que concreta las necesidades que poseen los estudiantes con determinada formación en un limitado espacio de tiempo, con conocimientos definidos, habilidades en el idioma inglés y valores 
para actuar en un contexto social (Sánchez, 2007).

\section{Metodología}

Se aplicó el método analítico sintético, el cual permitió analizar las informaciones teóricas con las que los docentes han ido trabajando en todo este tiempo hasta la actualidad (Chacón \& Herrera, 2010). Además, fue muy importante tomar en consideración el método de modelación, el cual fue utilizado para lograr una representación de la concepción preliminar de la estrategia metodológica a impartirse (Pardo et al., 2016).

Sobre la base de la revisión bibliográfica se aplicó el método analítico sintético, realizado mediante la selección de 15 documentos relevantes que aportan a la investigación, seleccionando 6 artículos de alto impacto sobre las estrategias metodológicas interactivas, obtenido así 15 estrategias metodológicas interactivas aplicadas actualmente en instituciones de educación superior, estrategias que se centran en potenciar tanto la enseñanza como el aprendizaje.

A su vez, es importante considerar que existen diferentes métodos de enseñanza utilizados en el aula dependiendo de la naturaleza de la asignatura, las instalaciones disponibles y el número de estudiantes (Ver Figura 1).

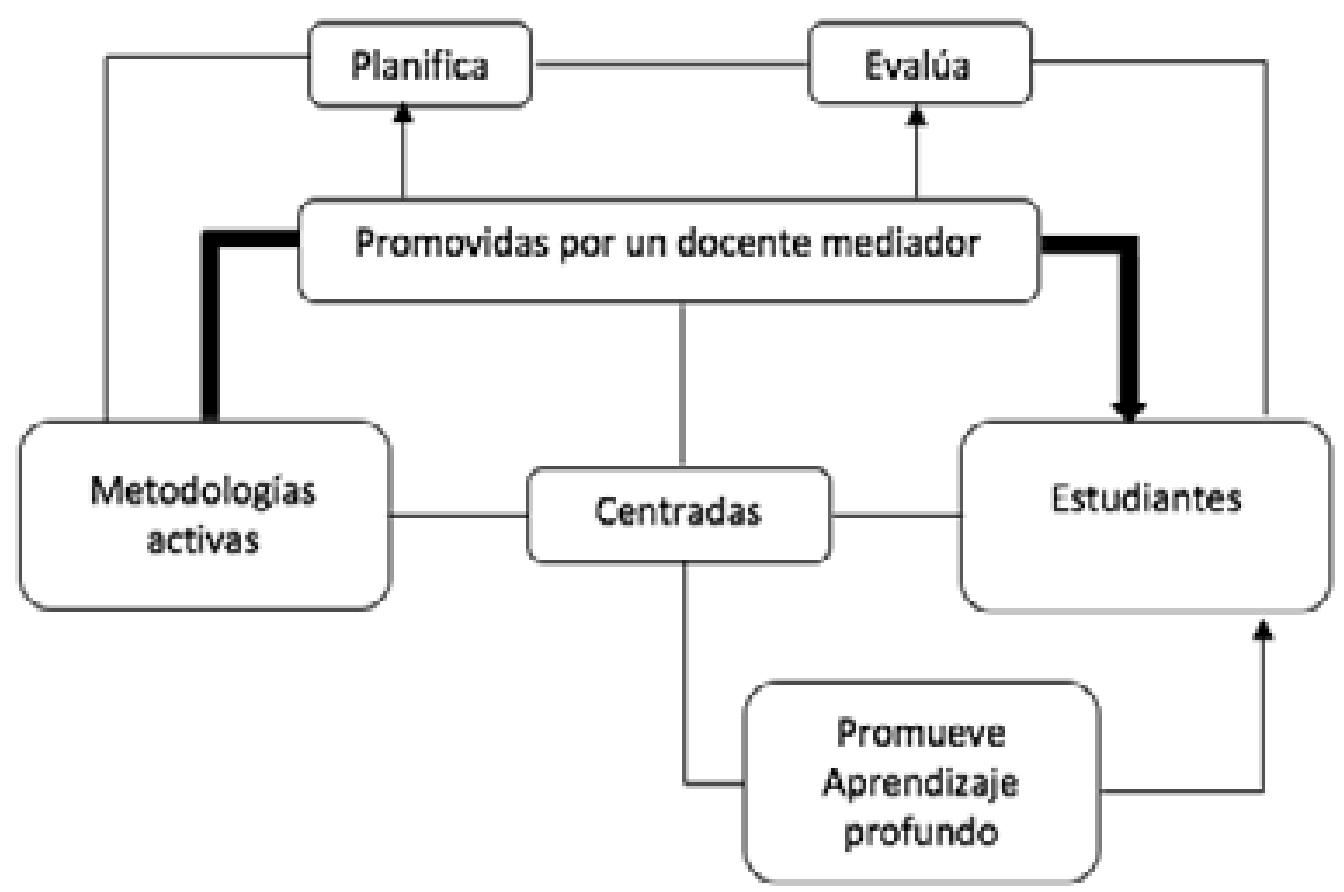

Figura 1. Metodologías

Fuente: Información obtenida de "Una propuesta de modelo para introducir metodologías activas en educación superior" Silva \& Maturana Castillo (2017) 


\section{Resultados}

En función a una revisión bibliográfica se determinó que, para la aplicación de estrategias metodológicas interactivas para la enseñanza y aprendizaje en la educación superior, esta centra en la forma de promover el aprendizaje por parte del docente

En la Tabla 1 se evidencian las actividades que los docentes tomaron en consideración para trabajar con sus estudiantes.

Tabla 1.

Actividades metodológicas

\begin{tabular}{ll}
\hline Actividades & \\
\hline Vacíos de información & Intercambio de información no conocida \\
\hline Discusiones y debates & $\begin{array}{l}\text { Comunicación oral donde se expone un tema y una } \\
\text { problemática }\end{array}$ \\
\hline $\begin{array}{l}\text { Enseñanza } \\
\text { contextualizada }\end{array}$ & Elección de contexto según la necesidad de los estudiantes \\
\hline Tópicos interesantes & $\begin{array}{l}\text { Utilizar material que motive al estudiante a captar su } \\
\text { atención }\end{array}$ \\
\hline Inferencias & Establecer puntos de vista de un contexto dado \\
\hline Skimming & Realizar una lectura rápida y eficiente \\
\hline Scanning & Lectura detallada en busca de datos específicos \\
\hline Ayuda visual & $\begin{array}{l}\text { Imágenes útiles al momento que se escucha una palabra } \\
\text { desconocida }\end{array}$ \\
\hline Realia & Materiales que hacen que la clase sea más interesante \\
\hline
\end{tabular}

Fuente: elaboración propia

El enfoque basado en competencias en el sistema de educación superior está destinado a aumentar la atención a la formación efectiva y tecnológica de las competencias profesionales. Por lo tanto, daremos una visión general de los métodos de enseñanza modernos que están más extendidos en la literatura científica y metodológica bajo el enfoque de competencias.

Debido a las variaciones en la terminología, los métodos y las estrategias utilizadas, fue necesario fusionar algunos de estos para facilitar la síntesis ( Tabla 2).

Tabla 2.

Métodos de enseñanza

\begin{tabular}{lll}
\hline Método & Descripción & Función \\
\hline Formación & La capacitación es un método de enseñanza & La ventaja de la capacitación es \\
& que tiene como objetivo desarrollar habilidades & que garantiza la participación activa \\
& y conocimientos en cualquier campo mediante & de todos los estudiantes en el \\
& la realización de tareas, actividades o juegos & proceso de capacitación. \\
& secuenciales. & \\
\hline
\end{tabular}




\begin{tabular}{|c|c|c|}
\hline Introducción & $\begin{array}{l}\text { Esta etapa establece el tono para el trabajo } \\
\text { futuro, creando una atmósfera relajada y } \\
\text { democrática. Se lleva a cabo en cualquier forma o } \\
\text { por cualquier medio }\end{array}$ & $\begin{array}{l}\text { El maestro informa a los } \\
\text { estudiantes de sus expectativas } \\
\text { y esperanzas con respecto a la } \\
\text { próxima actividad }\end{array}$ \\
\hline Descubrir & $\begin{array}{l}\text { Activa al grupo para participar en la interacción y } \\
\text { desarrollar habilidades de comunicación }\end{array}$ & $\begin{array}{l}\text { Debe hacerse incluso si los } \\
\text { estudiantes ya se conocen }\end{array}$ \\
\hline $\begin{array}{l}\text { Determinación } \\
\text { del orden de la } \\
\text { formación }\end{array}$ & $\begin{array}{l}\text { Cuando todos los participantes hablan o escriben } \\
\text { sobre sus expectativas, el maestro siempre les } \\
\text { dice el procedimiento de capacitación }\end{array}$ & $\begin{array}{l}\text { Abordar las necesidades del } \\
\text { alumno }\end{array}$ \\
\hline $\begin{array}{l}\text { Método de estudio } \\
\text { de caso }\end{array}$ & $\begin{array}{l}\text { El método de estudio de caso es la capacitación } \\
\text { mediante la resolución de casos específicos }\end{array}$ & $\begin{array}{l}\text { La esencia de este método es un } \\
\text { análisis colectivo de una situación, } \\
\text { encontrar una solución y una } \\
\text { defensa pública de dicha solución }\end{array}$ \\
\hline $\begin{array}{l}\text { Modelado de } \\
\text { comportamiento }\end{array}$ & $\begin{array}{l}\text { El modelado conductual es un método para } \\
\text { enseñar habilidades interpersonales y conducta } \\
\text { profesional. }\end{array}$ & $\begin{array}{l}\text { El método se lleva a cabo en } \\
\text { la siguiente secuencia: 1) la } \\
\text { presentación de un modelo de } \\
\text { comportamiento profesional que se } \\
\text { debe aprender; } 2 \text { ) la reproducción } \\
\text { más precisa del modelo de } \\
\text { comportamiento propuesto; y 3) } \\
\text { retroalimentación, que indica el } \\
\text { grado de éxito de dominar los } \\
\text { comportamientos relevantes. }\end{array}$ \\
\hline $\begin{array}{l}\text { El método de } \\
\text { retroalimentación } \\
\text { entre pares }\end{array}$ & $\begin{array}{l}\text { El método de retroalimentación entre pares es } \\
\text { cuando un alumno proporciona retroalimentación } \\
\text { continua a otro alumno sobre sus acciones, } \\
\text { acciones y decisiones }\end{array}$ & $\begin{array}{l}\text { Una mirada a sus acciones y } \\
\text { reacciones desde el exterior permite } \\
\text { al futuro especialista comprender } \\
\text { mejor sus fortalezas y debilidades } \\
\text { y desarrollar una autoestima } \\
\text { adecuada. }\end{array}$ \\
\hline
\end{tabular}

Proyectos de Play projects es un método de enseñanza donde juego el aprendizaje se efectúa mediante la resolución de problemas.
En la primera etapa, el profesor soluciona el problema de aprendizaje (investigación), es decir, hace que la situación del problema sea psicológica. En la segunda etapa, los estudiantes se dividieron en dos grupos competidores y elaboraron soluciones al problema. La tercera etapa es una reunión final donde los estudiantes toman roles y defienden públicamente las soluciones desarrolladas (revisadas por pares antes de la defensa).

El objetivo principal de un juego de metáforas es encontrar una nueva forma de resolver un problema dentro de la metáfora dada.

El profesor analiza la información recibida de los estudiantes, ofrece una solución alternativa, destaca las oportunidades perdidas, predice los resultados de las decisiones y hace recomendaciones para el futuro.

\begin{tabular}{lll}
\hline $\begin{array}{l}\text { Juego de la } \\
\text { metáfora }\end{array}$ & $\begin{array}{l}\text { El juego de metáforas es un método } \\
\text { de enseñanza destinado a desarrollar } \\
\text { nuevas actividades y cambiar actitudes de } \\
\text { comportamiento. }\end{array}$ & $\begin{array}{l}\text { El objetivo principal de un juego de } \\
\text { metáforas es encontrar una nueva } \\
\text { forma de resolver un problema } \\
\text { dentro de la metáfora dada. }\end{array}$ \\
\hline Método de cesta & El método de canasta es un método de & El profesor analiza la información \\
& $\begin{array}{l}\text { aprendizaje basado en la imitación de la } \\
\text { situación más común de especialistas, cuando } \\
\text { el estudiante tiene que realizar actividades no } \\
\text { planificadas de manera eficiente. }\end{array}$ & $\begin{array}{l}\text { recibida de los estudiantes, ofrece } \\
\text { las oportunidades perdidas, predice } \\
\end{array}$ \\
& $\begin{array}{l}\text { los resultados de las decisiones } \\
\text { y hace recomendaciones para el } \\
\text { futuro. }\end{array}$ \\
\end{tabular}




\begin{tabular}{ll}
\hline El método de & El método de aprendizaje activo se ha convertido \\
aprendizaje de & $\begin{array}{l}\text { recientemente en una de las áreas prometedoras } \\
\text { de la educación moderna, ya que proporciona la } \\
\text { acción }\end{array}$ \\
organización de entornos de autoaprendizaje.
\end{tabular}

Fuente: elaboración propia
El método de aprendizaje activo se ha convertido recientemente en una de las áreas prometedoras de la educación moderna, ya que proporciona la organización de entornos de autoaprendizaje.

Tabla 3.

Estrategias Metodológicas interactivas

\begin{tabular}{|c|c|c|c|c|c|c|}
\hline \multicolumn{7}{|c|}{ ESTRATEGIAS METODOLÓGICAS INTERACTIVAS } \\
\hline $\begin{array}{l}\text { Articulo } \\
\text { Estrategias }\end{array}$ & $\begin{array}{l}\text { Innovative } \\
\text { and } \\
\text { interactive } \\
\text { teaching } \\
\text { methods for } \\
\text { improving } \\
\text { learning } \\
\text { quality } \\
\text { in higher } \\
\text { education }\end{array}$ & $\begin{array}{l}\text { Estrategias } \\
\text { de } \\
\text { enseñanza }\end{array}$ & $\begin{array}{l}\text { Scrum } \\
\text { Methodology } \\
\text { in Higher } \\
\text { Education: } \\
\text { Innovation in } \\
\text { Teaching, } \\
\text { Learning and } \\
\text { Assessment }\end{array}$ & $\begin{array}{l}\text { E-Learning } \\
\text { Methodologies, } \\
\text { Strategies } \\
\text { and Tools to } \\
\text { implement } \\
\text { lifetime } \\
\text { education } \\
\text { anywhere } \\
\text { anytime }\end{array}$ & $\begin{array}{l}\text { Aprendizaje } \\
\text { mejorado por } \\
\text { la tecnología } \\
\text { o aprendizaje } \\
\text { impulsado por la } \\
\text { tecnología }\end{array}$ & $\begin{array}{l}\text { Una propuesta } \\
\text { de modelo } \\
\text { para introducir } \\
\text { metodologías } \\
\text { activas en } \\
\text { educación superior }\end{array}$ \\
\hline Métodos de lectura & $\mathrm{x}$ & & & & & \\
\hline Grupo de discusión & $x$ & & & & & \\
\hline Presentación individual & $\mathrm{X}$ & & & & & $\mathrm{x}$ \\
\hline Workshops & $\mathrm{x}$ & & & & & $x$ \\
\hline Role play & $x$ & & & & $x$ & \\
\hline Caso de estudio & & $x$ & $x$ & & & \\
\hline Conferencia & & $\mathrm{x}$ & & & & \\
\hline Discusión & & $\mathrm{x}$ & & & $x$ & \\
\hline Scrum projects & & & $\mathrm{x}$ & & & \\
\hline Intervenciones & & & $x$ & & & \\
\hline Análisis documental & & & $\mathrm{x}$ & & & \\
\hline E-Learning & & & & $\mathrm{x}$ & $x$ & \\
\hline $\begin{array}{l}\text { Aprendizaje } \\
\text { personalizado }\end{array}$ & & $\mathrm{x}$ & & & $\mathrm{x}$ & $\mathrm{x}$ \\
\hline $\begin{array}{l}\text { Intervención por } \\
\text { plataformas digitales }\end{array}$ & & & & & $\mathrm{x}$ & \\
\hline
\end{tabular}


Fuente: elaboración propia

En la Tabla 3 se presentan las diferentes estrategias metodológicas interactivas utilizadas, entre las cuales se destacan las interacciones, grupos de trabajo, análisis discusiones, entre otras., las mismas que aportaron para conocer las principales estrategias de enseñanza y aprendizaje. Estas características en cada uno de los documentos revisados determinaron que en los últimos años las tácticas aplicadas en la educación superior son diversas y de relevancia participativas y vivenciales.

Para concluir la revisión, observamos que los métodos de aprendizaje activo modifican el rol del maestro desde el traductor de información hasta el organizador y coordinador del proceso educativo y permiten formar competencias complejas en futuras especialidades profesionales a través de actividades estudiantiles que se manifiestan de manera tan cercana como sea posible el contenido del trabajo profesional.

\section{Conclusiones}

En las estrategias más utilizadas y recomendadas se encuentra el role play, caso de estudio, aprendizaje personalizado, discusiones y E-learning, herramientas que se generan en función de los módulos y necesidades de enseñanza, tomando en cuenta que gran parte del aprendizaje universitario es de modalidad autónoma, a pesar de ello, la guía de un docente es el punto más importante a considerar, es por eso que se destacan tanto actividades individuales como estrategias de formación grupal.

Entre las técnicas más innovadoras y representativas se diferencia scrum projects y la intervención por plataformas digitales, la primera mencionada se centra en el aprendizaje de los estudiantes de acuerdo a los intereses personales, generando proyectos de interés estudiantil y la segunda se encarga de la aplicación de herramientas digitales como medios de gestión académica.

Con respecto a la metodología de enseñanza no se desarrolla de acuerdo a las necesidades del estudiante en cambio se lleva un módulo de acuerdo al nivel en que se encuentran. La tecnología se considera como importante pero no se utiliza por el costo y por el grado de dificultad que puede generar para los docentes al momento de poder impartir a sus estudiantes. Para finalizar, podemos decir que el aprendizaje colaborativo existe ya que los estudiantes como los docentes dan todo de ellos para poder entender y enseñar. 


\section{Referencias}

Bonilla, D., Hallo, D., Quizhpe, G., \& Taco, C. (2020). El comportamiento escolar basado en la relación parental en estudiantes de idioma extranjero. Revista Científica UISRAEL, 7(1), 137-152.

Carrera, C., \& Marín, R. (2011). Modelo pedagógico para el desarrollo de competencias en educación superior. Actualidades Investigativas En Educación, 11(1), 1-32. https://doi.org/10.15517/aie.v11i1.10183

Chacón, A., \& Herrera, M. (2010). Estrategias metodológicas utilizadas por los docentes en el proceso enseñanza aprendizaje de vocabulario en contexto del inglés en los primeros y segundos años de bachillerato del colegio técnico nacional Mariano Suárez Veintimilla. In Universidad Técnica del Norte (Vol. 9, Issue 1).

Granjeiro, É. (2019). Research-based teaching-learning method: a strategy to motivate and engage students in human physiology classes. Advances in Physiology Education, 43, 553-556. https://doi.org/10.1152/ advan.00034.2019

Gutiérrez, J., Jacob, F., \& Gutiérrez, C. (2018). Estrategias didácticas de enseñanza y aprendizaje desde una perspectiva interactiva. Journal of Chemical Information and Modeling, 53(9), 1689-1699. https://doi. org/10.1017/CBO9781107415324.004

Jurado, A., \& Munoz, R. (2017). Scrum Methodology in Higher Education: Innovation in Teaching, Learning and Assessment. International Journal of Higher Education, 6(6), 1-18.

Karmakar, A., \& Nath, A. (2014). E-Learning Methodologies, Strategies and Tools to implement lifetime education anywhere anytime. International Journal of Innovative Research in Advanced Engineering, 1(4), $193-201$.

Lara, A., Lara, N., Lara, G., \& Bonilla, D. (2018). Aprendizaje basado en problemas como estrategia significativa en la formación académica. Universidad Ciencia y Tecnología, (1), 8-8.

Ledesma, L. P., \& Labrador, E. L. (2010). Propuesta de estrategia metodológica para la asignatura de inglés en estudiantes de 4to año de Medicina. Revista de Ciencias Médicas de Pinar Del Río, 14(1), 327-338.

Mora, E., Bonilla, D., Núñez, L., \& Sarmiento, J. (2018). Inadaptabilidad de los docentes al manejo de plataformas virtuales: caso educarecuador. Revista Pedagógica de La Universidad de Cienfuegos, 14(62), 1-5. http://scielo.sld.cu/pdf/rc/v14n62/rc066218.pdf

Pardo, J., Mora, N., \& Guzmán, M. (2016). Estrategias didácticas para la enseñanza del inglés en primero y segundo de primaria 1. In Universidad de la Salle.

Petruta, G. (2013). Teacher's Opinion on the use of Interactive Methods/Techniques in Lessons. Procedia - Social and Behavioral Sciences, 76, 649-653. https://doi.org/10.1016/j.sbspro.2013.04.180 
Sánchez, D. (2007). Estrategias didácticas para el perfeccionamiento del proceso de enseñanza - aprendizaje acelerado del idioma inglés en los cursos básicos para profesionales de la salud. In Instituto superior pedagógico Felix Varela.

Silva, J., \& Maturana Castillo, D. (2017). Una propuesta de modelo para introducir metodologías activas en educación superior. Innovación Educativa, 17(73), 117-131.

Wickramsinghe, S. (2016). Innovative and interactive teaching methods for improving learning quality in higher education. 2nd International Conference on Education and Distance Learning, 1-12.

Yakovleva, N., \& Yakovlev, E. (2014). Interactive teaching methods in contemporary higher education. Pacific Science Review, 16(2), 75-80. https://doi.org/10.1016/j.pscr.2014.08.016 
Copyright (c) 2020 María de los Ángeles Bonilla, Jonathan Patricio Cárdenas Benavides, Florcita Janeth Arellano Espinoza y Danny Fernando Pérez Castillo

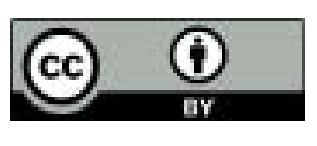

Este texto está protegido bajo una licencia internacional Creative Commons 4.0.

Usted es libre para Compartir-copiar y redistribuir el material en cualquier medio o formato

- y Adaptar el documento - remezclar, transformar y crear a partir del material-para cualquier propósito, incluso para fines comerciales, siempre que cumpla las condiciones de Atribución. Usted debe dar crédito a la obra original de manera adecuada, proporcionar un enlace a la licencia, e indicar si se han realizado cambios. Puede hacerlo en cualquier forma razonable, pero no de forma tal que sugiera que tiene el apoyo del licenciante o lo recibe por el uso que hace de la obra.

\section{$\underline{\text { Resumen de licencia - Texto completo de la licencia }}$}

